\title{
Roughness of Filters in Lattice Implication Algebras
}

\author{
by \\ Young Bae JUN and Yang XU \\ Presented by Zdzistaw PAWLAK
}

Summary. As a generalization of filters in lattice implication algebras, the notion of rough filters in lattice implication algebras is introduced, and some of their properties are considered.

1. Introduction. Various theories and methods have been proposed to deal with incomplete and insufficient information in classification, concept formation, and data analysis in data mining. For example, fuzzy set theory [26], rough sets [19], computing with words [21, 27, 28], linguistic dynamic systems [20, 21], and many others, have been developed and applied to real-world problems.

The concept of a rough set was originally proposed by Pawlak $[18,19]$ as a formal tool for modelling and processing incomplete information in information systems. Rough set theory has been conceived as a tool to conceptualize, organize and analyze various types of data, in particular, to deal with inexact, uncertain or vague knowledge in applications related to artificial intelligence. The algebraic approach to rough sets was studied in $[1,3$, $8,11,12,13]$.

The theory of rough sets is an extension of set theory in which a subset of a universe is described by a pair of ordinary sets called the lower and upper approximations. A key notion in the rough set model is an equivalence relation. The equivalence classes are the building blocks for the construction of the lower and upper approximations. The lower approximation of a given set is the union of all the equivalence classes which are subsets of the set, and

2000 Mathematics Subject Classification: 03G25, 06D05, 06D99.

Key words and phrases: lattice implication algebra, filter, rough filter, implication homomorphism, dual kernel. 
the upper approximation is the union of all the equivalence classes which have a nonempty intersection with the set.

In the field of many-valued logic, lattice-valued logic plays an important role in two aspects. One is that it extends the chain-type truth-value field of some well known presented logic [2] to some relatively general lattices. The other is that the incomplete comparability of truth values characterized by a general lattice can more efficiently reflect the uncertainty of human thinking, judgment and decision. Hence, lattice-valued logic is becoming a research field which strongly influences the development of algebraic logic, computer science and artificial intelligence technology. Therefore Goguen [4], Pavelka [17] and Novak [16] researched lattice-valued logic formal systems. Moreover, in order to establish a logic system with truth values in a relatively general lattice, in 1990, during the work on the project "The Study of Abstract Fuzzy Logic" supported by National Natural Science Foundation in China, $\mathrm{Xu}$ introduced lattice implication algebras by combining lattice algebras and implication algebras, and investigated many useful structures $[14,15$, $23,24]$. Lattice implication algebras provided a foundation for establishing the corresponding logic system from the algebraic viewpoint. For the general development of lattice implication algebras, filter theory plays an important role (see $[5,6,7,9,10,24])$.

In this paper, we apply rough set theory to lattice implication algebras, and we introduce the notion of upper/lower rough filters, which extends the notion of a filter in a lattice implication algebra.

2. Preliminaries. By a lattice implication algebra we mean a bounded lattice $(L, \vee, \wedge, 0,1)$ with order-reversing involution ")" and a binary operation " $\rightarrow$ " satisfying the following axioms:

(I1) $x \rightarrow(y \rightarrow z)=y \rightarrow(x \rightarrow z)$,

(I2) $x \rightarrow x=1$,

(I3) $x \rightarrow y=y^{\prime} \rightarrow x^{\prime}$,

(I4) $x \rightarrow y=y \rightarrow x=1 \Rightarrow x=y$,

(I5) $(x \rightarrow y) \rightarrow y=(y \rightarrow x) \rightarrow x$,

(L1) $(x \vee y) \rightarrow z=(x \rightarrow z) \wedge(y \rightarrow z)$,

(L2) $(x \wedge y) \rightarrow z=(x \rightarrow z) \vee(y \rightarrow z)$,

for all $x, y, z \in L$. For any lattice implication algebra $L$, the relation $\leq$ defined by $x \leq y$ if and only if $x \rightarrow y=1$ is a partial order on $L$. In a lattice implication algebra $L$, the following hold (see [23]):

(a1) $0 \rightarrow x=1,1 \rightarrow x=x$ and $x \rightarrow 1=1$.

(a2) $x \rightarrow y \leq(y \rightarrow z) \rightarrow(x \rightarrow z)$.

(a3) $x \leq y$ implies $y \rightarrow z \leq x \rightarrow z$ and $z \rightarrow x \leq z \rightarrow y$.

(a4) $x^{\prime}=x \rightarrow 0$. 
(a5) $x \vee y=(x \rightarrow y) \rightarrow y$.

(a6) $\left((y \rightarrow x) \rightarrow y^{\prime}\right)^{\prime}=x \wedge y=\left((x \rightarrow y) \rightarrow x^{\prime}\right)^{\prime}$.

(a7) $x \leq(x \rightarrow y) \rightarrow y$.

A nonempty subset $S$ of a lattice implication algebra $L$ is said to be a quasi-subalgebra of $L$ if $x \rightarrow y \in S$ whenever $x, y \in S$. A nonempty subset $F$ of a lattice implication algebra $L$ is called a filter of $L$ if it satisfies

- $1 \in F$,

- $(\forall x \in F)(\forall y \in L)(x \rightarrow y \in F \Rightarrow y \in F)$.

Let $L_{1}$ and $L_{2}$ be lattice implication algebras. A mapping $f: L_{1} \rightarrow L_{2}$ is called an implication homomorphism ([22]) if $f(x \rightarrow y)=f(x) \rightarrow f(y)$ for all $x, y \in L_{1}$. Moreover, if $f$ satisfies the conditions

$$
f(x \vee y)=f(x) \vee f(y), \quad f(x \wedge y)=f(x) \wedge f(y), \quad f\left(x^{\prime}\right)=(f(x))^{\prime}
$$

for all $x, y \in L_{1}$, we say that $f$ is a lattice implication homomorphism. Note that if an implication homomorphism $f: L_{1} \rightarrow L_{2}$ satisfies $f(0)=0$, then $f$ is a lattice implication homomorphism ([22]).

3. Rough sets in lattice implication algebras. In what follows let $L$ denote a lattice implication algebra unless otherwise specified.

An equivalence relation $\varrho$ on $L$ is called a congruence relation on $L$ if whenever $(x, y),(u, v) \in \varrho$ then $(x \rightarrow u, y \rightarrow v) \in \varrho$. We denote by $[a]_{\varrho}$ the $\varrho$-congruence class containing the element $a \in L$. Let $L / \varrho$ denote the set of all $\varrho$-congruence classes on $L$, i.e., $L / \varrho:=\left\{[a]_{\varrho} \mid a \in L\right\}$. For any $[x]_{\varrho},[y]_{\varrho} \in L / \varrho$, if we define $[x]_{\varrho} \rightarrow[y]_{\varrho}=[x \rightarrow y]_{\varrho},[x]_{\varrho} \cup[y]_{\varrho}=[x \vee y]_{\varrho}$, $[x]_{\varrho} \cap[y]_{\varrho}=[x \wedge y]_{\varrho}$, and $[x]_{\varrho}^{\prime}=\left[x^{\prime}\right]_{\varrho}$, then $\left(L / \varrho, \cap, \cup,{ }^{\prime}, \rightarrow\right)$ is a lattice implication algebra (see [25]).

Let $\varrho$ be an equivalence relation on $L$ and let $\mathcal{P}(L)$ denote the power set of $L$ and $\mathcal{P}^{*}(L)=\mathcal{P}(L) \backslash\{\emptyset\}$. For all $x \in L$, let $[x]_{\varrho}$ denote the equivalence class of $x$ with respect to $\varrho$. Define the functions $\varrho_{*}, \varrho^{*}: \mathcal{P}(L) \rightarrow \mathcal{P}(L)$ as follows: $\forall S \in \mathcal{P}(L)$,

$$
\varrho_{*}(S)=\left\{x \in L \mid[x]_{\varrho} \subseteq S\right\}, \quad \varrho^{*}(S)=\left\{x \in L \mid[x]_{\varrho} \cap S \neq \emptyset\right\} .
$$
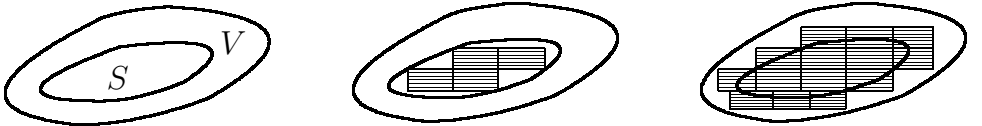

$\varrho_{*}(S)$ is called the $\varrho$-lower approximation of $S$ while $\varrho^{*}(S)$ is called the $\varrho$-upper approximation of $S$. For a nonempty subset $S$ of $L$,

$$
\varrho(S)=\left(\varrho_{*}(S), \varrho^{*}(S)\right)
$$

is called a rough set with respect to $\varrho$ of $\mathcal{P}(L) \times \mathcal{P}(L)$ if $\varrho_{*}(S) \neq \varrho^{*}(S)$. 
A subset $S$ of $L$ is said to be definable if $\varrho_{*}(S)=\varrho^{*}(S)$. The pair $(L, \varrho)$ is called an approximation space.

The following property is useful for our research (cf. [18]).

Proposition 3.1. Let $\varrho$ and $\lambda$ be congruence relations on L. Then:

(1) $\left(\forall F \in \mathcal{P}^{*}(L)\right)\left(\varrho_{*}(F) \subseteq F \subseteq \varrho^{*}(F)\right)$.

(2) $\left(\forall F, G \in \mathcal{P}^{*}(L)\right)\left(\varrho^{*}(F \cup G)=\varrho^{*}(F) \cup \varrho^{*}(G)\right)$.

(3) $\left(\forall F, G \in \mathcal{P}^{*}(L)\right)\left(\varrho_{*}(F \cap G)=\varrho_{*}(F) \cap \varrho_{*}(G)\right)$.

(4) $\left(\forall F, G \in \mathcal{P}^{*}(L)\right)\left(F \subseteq G \Rightarrow \varrho_{*}(F) \subseteq \varrho_{*}(G)\right)$.

(5) $\left(\forall F, G \in \mathcal{P}^{*}(L)\right)\left(F \subseteq G \Rightarrow \varrho^{*}(F) \subseteq \varrho^{*}(G)\right)$.

(6) $\left(\forall F, G \in \mathcal{P}^{*}(L)\right)\left(\varrho_{*}(F) \cup \varrho_{*}(G) \subseteq \varrho_{*}(F \cup G)\right)$.

(7) $\left(\forall F, G \in \mathcal{P}^{*}(L)\right)\left(\varrho^{*}(F \cap G) \subseteq \varrho^{*}(F) \cap \varrho^{*}(G)\right)$.

(8) $\left(\forall F \in \mathcal{P}^{*}(L)\right)\left(\varrho \subseteq \lambda \Rightarrow \lambda_{*}(F) \subseteq \varrho_{*}(F), \varrho^{*}(F) \subseteq \lambda^{*}(F)\right)$.

Proof. Straightforward.

COROLlaRY 3.2. If $\varrho$ and $\lambda$ are congruence relations on $L$, then:

(i) $\left(\forall F \in \mathcal{P}^{*}(L)\right)\left((\varrho \cap \lambda)^{*}(F) \subseteq \varrho^{*}(F) \cap \lambda^{*}(F)\right)$.

(ii) $\left(\forall F \in \mathcal{P}^{*}(L)\right)\left(\varrho_{*}(F) \cap \lambda_{*}(F) \subseteq(\varrho \cap \lambda)_{*}(F)\right)$.

Proof. This follows immediately from Proposition 3.1.

For any $F, G \in \mathcal{P}^{*}(L)$, we define $F \rightarrow G:=\{a \rightarrow b \mid a \in F, b \in G\}$.

THEOREM 3.3. If $\varrho$ is a congruence relation on $L$, then

$$
\left(\forall F, G \in \mathcal{P}^{*}(L)\right)\left(\varrho^{*}(F) \rightarrow \varrho^{*}(G) \subseteq \varrho^{*}(F \rightarrow G)\right) .
$$

Proof. Let $c \in \varrho^{*}(F) \rightarrow \varrho^{*}(G)$. Then there exist $a \in \varrho^{*}(F)$ and $b \in \varrho^{*}(G)$ such that $c=a \rightarrow b$. It follows that $x \in[a]_{\varrho} \cap F$ and $y \in[b]_{\varrho} \cap G$ for some $x, y \in L$. Hence $x \rightarrow y \in[a]_{\varrho} \rightarrow[b]_{\varrho}=[a \rightarrow b]_{\varrho}$ and $x \rightarrow y \in F \rightarrow G$, that is, $x \rightarrow y \in[a \rightarrow b]_{\varrho} \cap(F \rightarrow G)$. Thus $c=a \rightarrow b \in \varrho^{*}(F \rightarrow G)$, and so $\varrho^{*}(F) \rightarrow \varrho^{*}(G) \subseteq \varrho^{*}(F \rightarrow G)$.

THEOREM 3.4. If $\varrho$ is a congruence relation on $L$, then

$\left(\forall F, G \in \mathcal{P}^{*}(L)\right)\left(\varrho_{*}(F \rightarrow G) \neq \emptyset \Rightarrow \varrho_{*}(F) \rightarrow \varrho_{*}(G) \subseteq \varrho_{*}(F \rightarrow G)\right)$.

Proof. Let $c \in \varrho_{*}(F) \rightarrow \varrho_{*}(G)$. Then $c=a \rightarrow b$ for some $a \in \varrho_{*}(F)$ and $b \in \varrho_{*}(G)$. Thus we get $[a]_{\varrho} \subseteq F$ and $[b]_{\varrho} \subseteq G$. It follows that

$$
[a \rightarrow b]_{\varrho}=[a]_{\varrho} \rightarrow[b]_{\varrho} \subseteq F \rightarrow G
$$

so that $c=a \rightarrow b \in \varrho_{*}(F \rightarrow G)$. Therefore the result is valid.

The following example shows that the condition $\varrho_{*}(F \rightarrow G) \neq \emptyset$ in Theorem 3.4 is necessary.

Example 3.5. Let $L:=\{0, a, b, c, 1\}$. Define the partial order on $L$ as $0 \leq a \leq b \leq c \leq 1$, and define

$$
x \wedge y:=\min \{x, y\}, \quad x \vee y:=\max \{x, y\}
$$


for all $x, y \in L$, and "\%" and " $\rightarrow$ " as follows:

\begin{tabular}{l|l}
$x$ & $x^{\prime}$ \\
\hline 0 & 1 \\
$a$ & $c$ \\
$b$ & $b$ \\
$c$ & $a$ \\
1 & 0
\end{tabular}

\begin{tabular}{c|ccccc}
$\rightarrow$ & 0 & $a$ & $b$ & $c$ & 1 \\
\hline 0 & 1 & 1 & 1 & 1 & 1 \\
$a$ & $c$ & 1 & 1 & 1 & 1 \\
$b$ & $b$ & $c$ & 1 & 1 & 1 \\
$c$ & $a$ & $b$ & $c$ & 1 & 1 \\
1 & 0 & $a$ & $b$ & $c$ & 1
\end{tabular}

Then $(L, \vee, \wedge, \prime, \rightarrow)$ is a lattice implication algebra (see [24]). Consider a congruence relation $\varrho$ on $L$ with the following $\varrho$-congruence classes:

$$
\{0\},\{a\},\{b\},\{c, 1\} .
$$

Taking $F=\{0, a\}$ and $G=\{b\}$, we have $F \rightarrow G=\{1\}, \varrho_{*}(F \rightarrow G)=\emptyset$, $\varrho_{*}(F)=\{0, a\}, \varrho_{*}(G)=\{b\}$, and $\varrho_{*}(F) \rightarrow \varrho_{*}(G)=\{1\}$.

For any congruence relation $\varrho$ on $L$, we note that

- $\left(\forall F \in \mathcal{P}^{*}(L)\right)\left(\varrho_{*}(F) \subseteq F\right)$,

- $\left(\forall F, G \in \mathcal{P}^{*}(L)\right)\left(F \subseteq G \Rightarrow \varrho_{*}(F) \subseteq \varrho_{*}(G)\right)$,

- $\left(\forall F \in \mathcal{P}^{*}(L)\right)\left(\varrho_{*}\left(\varrho_{*}(F)\right)=\varrho_{*}(F)\right)$,

which means that $\varrho_{*}$ is an interior operator on $L$. This operation induces a topology $\mathcal{T}$ on $L$ such that

$$
F \in \mathcal{T} \Leftrightarrow \varrho_{*}(F)=F .
$$

LEMMA 3.6. For any congruence relation $\varrho$ on $L, \varrho^{*}$ is a closure operator on the topological space $(L, \mathcal{T})$.

Proof. For any $F \in \mathcal{P}^{*}(L)$ we have $x \in \varrho^{*}(F) \Leftrightarrow[x]_{\varrho} \cap F \neq \emptyset \Leftrightarrow[x]_{\varrho} \nsubseteq F^{\mathrm{c}} \Leftrightarrow x \notin \varrho_{*}\left(F^{\mathrm{c}}\right) \Leftrightarrow x \in\left(\varrho_{*}\left(F^{\mathrm{c}}\right)\right)^{\mathrm{c}}$, that is, $\varrho^{*}(F)=\left(\varrho_{*}\left(F^{\mathrm{c}}\right)\right)^{\mathrm{c}}$, which completes the proof.

LEMMA 3.7. For any congruence relation $\varrho$ on $L$, we have

(i) $(\forall F \in \mathcal{P}(L))\left(\varrho_{*}(F)=F \Leftrightarrow \varrho^{*}\left(F^{\mathrm{c}}\right)=F^{\mathrm{c}}\right)$.

(ii) $(\forall F \in \mathcal{P}(L))\left(\varrho_{*}(F)=F \Leftrightarrow \varrho^{*}(F)=F\right)$.

Proof. Straightforward.

Based on the above two lemmas we have the following result.

THEOREM 3.8. For any $F \subseteq L$ and a congruence relation $\varrho$ on $L$, the following assertions are equivalent:

(i) $F$ is definable with respect to $\varrho$.

(ii) $F$ is open in the topological space $(L, \mathcal{T})$.

(iii) $F$ is closed in the topological space $(L, \mathcal{T})$. 
According to [12], we say that an open set $F$ of $L$ is free in the approximation space $(L, \varrho)$ if $x \notin \varrho^{*}(F \backslash\{x\})$ for all $x \in L$. Since $\varrho^{*}(F \backslash\{x\})=\left(\varrho_{*}((F \backslash\right.$ $\left.\left.\{x\})^{\mathrm{c}}\right)\right)^{\mathrm{c}}$, a nonempty subset $F$ of $L$ is free if and only if $x \in \varrho_{*}\left(F^{\mathrm{c}} \cup\{x\}\right)$, i.e., if and only if $[x]_{\varrho} \subseteq F^{\mathrm{c}} \cup\{x\}$ for every $x \in F$. Thus for a free subset $F$ and any $(x, y) \in \varrho \cap(F \times F)$ we have $y \in F$, which together with $y \in[x]_{\varrho} \subseteq F^{\mathrm{c}} \cup\{x\}$ implies that $y=x$. Therefore $\varrho \cap(F \times F)=\{(a, a) \mid a \in F\}$. Conversely, let $\varrho \cap(F \times F)=\{(a, a) \mid a \in F\}$ and let $y$ be an arbitrary element of $[x]_{\varrho}$. If $y \in F$, then $y=x$, i.e., $y \in\{x\} \subseteq F^{\mathrm{c}} \cup\{x\}$. If $y \notin F$, then $y \in F^{\mathrm{c}} \subseteq F^{\mathrm{c}} \cup\{x\}$. Thus, in each case $[x]_{\varrho} \subseteq F^{\mathrm{c}} \cup\{x\}$, which means that $F$ is free. Consequently, we obtain the following characterization of free subsets.

Theorem 3.9. $F \subseteq L$ is free if and only if $\varrho \cap(F \times F)=\{(a, a) \mid a \in F\}$.

COROLlary 3.10. If $L$ is free, then any subset of $L$ is free.

4. Rough filters in lattice implication algebras. Let $F$ be a filter of $L$. Define a relation $\Theta$ on $L$ by

$$
(x, y) \in \Theta \Leftrightarrow\{x \rightarrow y, y \rightarrow x\} \subseteq F .
$$

Then $\Theta$ is a congruence relation on $L$ related to the filter $F$ of $L$. Let $F_{x}$ denote the equivalence class of $L$ with respect to the equivalence relation $\Theta$ related to the filter $F$ of $L$, that is, $F_{x}=\{y \in L \mid\{x \rightarrow y, y \rightarrow x\} \subseteq F\}$, and $L / F$ denote the collection of all equivalence classes, that is, $L / F=$ $\left\{F_{x} \mid x \in L\right\}$. Then $F_{1}=F$. If we define binary operations $\sqcap, \sqcup, \rightsquigarrow$ and a unary operation ${ }^{\bullet}$ on $L / F$ as follows: $F_{x} \sqcup F_{y}=F_{x \vee y}, F_{x} \sqcap F_{y}=F_{x \wedge y}$, $F_{x} \rightsquigarrow F_{y}=F_{x \rightarrow y}, F_{x}^{\bullet}=F_{x^{\prime}}$, then $(L / F, \sqcup, \sqcap, \bullet, \rightsquigarrow)$ is a lattice implication algebra. Let $\Theta$ be an equivalence relation on $L$ related to a filter $F$ of $L$. For any nonempty subset $S$ of $L$, the lower and upper approximations of $S$ are denoted by $\underline{\Theta}(F ; S)$ and $\bar{\Theta}(F ; S)$ respectively, that is,

$$
\underline{\Theta}(F ; S)=\left\{x \in L \mid F_{x} \subseteq S\right\}, \quad \bar{\Theta}(F ; S)=\left\{x \in L \mid F_{x} \cap S \neq \emptyset\right\} .
$$

If $F=S$, then $\underline{\Theta}(F ; S)$ and $\bar{\Theta}(F ; S)$ are denoted by $\underline{\Theta}(F)$ and $\bar{\Theta}(F)$, respectively.

EXAmPle 4.1. (1) Let $L=\{0, a, b, 1\}$ be a set with Hasse diagram and Cayley tables as follows:

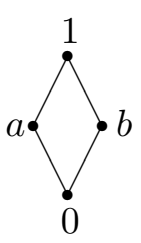

\begin{tabular}{l|l}
$x$ & $x^{\prime}$ \\
\hline 0 & 1 \\
$a$ & $b$ \\
$b$ & $a$ \\
1 & 0
\end{tabular}

\begin{tabular}{c|cccc}
$\rightarrow$ & 0 & $a$ & $b$ & 1 \\
\hline 0 & 1 & 1 & 1 & 1 \\
$a$ & $b$ & 1 & $b$ & 1 \\
$b$ & $a$ & $a$ & 1 & 1 \\
1 & 0 & $a$ & $b$ & 1
\end{tabular}

Define $\vee$ - and $\wedge$-operations on $L$ as follows:

$$
x \vee y=(x \rightarrow y) \rightarrow y, \quad x \wedge y=\left(\left(x^{\prime} \rightarrow y^{\prime}\right) \rightarrow y^{\prime}\right)^{\prime}
$$


for all $x, y \in L$. Then $\left(L, \vee, \wedge,{ }^{\prime}, \rightarrow\right)$ is a lattice implication algebra. It is easy to check that $F:=\{1, a\}$ and $G:=\{1, b\}$ are filters of $L$. Let $\Theta$ be an equivalence relation on $L$ related to $F$. Then $F_{0}=F_{b}=\{0, b\}$ and $F_{a}=F_{1}=A$. Hence, for every nonempty proper subset $S$ of $L$ we have

$$
\begin{aligned}
& \underline{\Theta}(F ; S)= \begin{cases}\{1, a\} & \text { if }\{1, a\} \subseteq S, \\
\{0, b\} & \text { if }\{0, b\} \subseteq S, \\
\emptyset & \text { otherwise, }\end{cases} \\
& \bar{\Theta}(F ; S)= \begin{cases}\{1, a\} & \text { if } S=\{1\},\{a\}, \text { or }\{1, a\}, \\
\{0, b\} & \text { if } S=\{0\},\{b\}, \text { or }\{0, b\}, \\
L & \text { otherwise. }\end{cases}
\end{aligned}
$$

(2) Let $L=\{0, a, b, c, d, 1\}$ be a set with Hasse diagram and Cayley tables as follows:

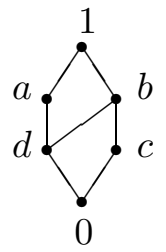

\begin{tabular}{l|l}
$x$ & $x^{\prime}$ \\
\hline 0 & 1 \\
$a$ & $c$ \\
$b$ & $d$ \\
$c$ & $a$ \\
$d$ & $b$ \\
1 & 0
\end{tabular}

\begin{tabular}{c|cccccc}
$\rightarrow$ & 0 & $a$ & $b$ & $c$ & $d$ & 1 \\
\hline 0 & 1 & 1 & 1 & 1 & 1 & 1 \\
$a$ & $c$ & 1 & $b$ & $c$ & $b$ & 1 \\
$b$ & $d$ & $a$ & 1 & $b$ & $a$ & 1 \\
$c$ & $a$ & $a$ & 1 & 1 & $a$ & 1 \\
$d$ & $b$ & 1 & 1 & $b$ & 1 & 1 \\
1 & 0 & $a$ & $b$ & $c$ & $d$ & 1
\end{tabular}

Then $\left(L, \vee, \wedge,^{\prime}, \rightarrow\right)$ is a lattice implication algebra and the set $F=\{b, c, 1\}$ is a filter of $L$ (see [9]). Let $\Theta$ be an equivalence relation on $L$ related to $F$. Then $F_{0}=F_{a}=F_{d}=\{0, a, d\}$ and $F_{b}=F_{c}=F_{1}=\{b, c, 1\}$. Hence, for every nonempty proper subset $S$ of $L$ we have

$\underline{\Theta}(F ; S)= \begin{cases}\{0, a, d\} & \text { if }\{0, a, d\} \subseteq S, \\ \{b, c, 1\} & \text { if }\{b, c, 1\} \subseteq S, \\ \emptyset & \text { otherwise, }\end{cases}$

$\bar{\Theta}(F ; S)= \begin{cases}\{0, a, d\} & \text { if } S=\{0\},\{a\},\{d\},\{0, a\},\{0, d\},\{a, d\} \text { or }\{0, a, d\} \\ \{b, c, 1\} & \text { if } S=\{1\},\{b\},\{c\},\{b, c\},\{b, 1\},\{c, 1\} \text { or }\{b, c, 1\} \\ L & \text { otherwise. }\end{cases}$

Let $\Theta$ be an equivalence relation on $L$ related to a filter $F$. We note that if $G$ is not a filter of $L$, then $\underline{\Theta}(F ; G)$ and $\bar{\Theta}(F ; G)$ are not filters of $L$. In Example 4.1, we see that there exists a nonfilter $U$ of $L$ such that $\underline{\Theta}(F ; U)$ is a filter of $L$, and there exists a nonfilter $V$ of $L$ such that $\bar{\Theta}(F ; V)$ is a filter of $L$.

Proposition 4.2. Let $\Theta$ and $\Psi$ be equivalence relations on $L$ related to filters $F$ and $G$ of $L$, respectively. If $F \subseteq G$, then $\Theta \subseteq \Psi$. 
Proof. If $(x, y) \in \Theta$, then $x \rightarrow y \in F \subseteq G$ and $y \rightarrow x \in F \subseteq G$. Hence $(x, y) \in \Psi$, and so $\Theta \subseteq \Psi$.

Proposition 4.3. Let $\Theta$ be an equivalence relation on $L$ related to a filter $F$ of $L$. Then:

(1) $(\forall S \in \mathcal{P}(L))(\underline{\Theta}(F ; S) \subseteq S \subseteq \bar{\Theta}(F ; S))$.

(2) $(\forall S, T \in \mathcal{P}(L))(\bar{\Theta}(F ; S \cup T)=\bar{\Theta}(F ; S) \cup \bar{\Theta}(F ; T))$.

(3) $(\forall S, T \in \mathcal{P}(L))(\underline{\Theta}(F ; S \cap T)=\underline{\Theta}(F ; S) \cap \underline{\Theta}(F ; T))$.

(4) $(\forall S, T \in \mathcal{P}(L))(S \subseteq T \Rightarrow \underline{\Theta}(F ; S) \subseteq \underline{\Theta}(F ; T), \bar{\Theta}(F ; S) \subseteq \bar{\Theta}(F ; T))$.

(5) $(\forall S, T \in \mathcal{P}(L))(\underline{\Theta}(F ; S \cup T) \supseteq \underline{\Theta}(F ; S) \cup \underline{\Theta}(F ; T))$.

(6) $(\forall S, T \in \mathcal{P}(L))(\bar{\Theta}(F ; S \cap T) \subseteq \bar{\Theta}(F ; S) \cap \bar{\Theta}(F ; T))$.

(7) If $\Psi$ is an equivalence relation on $L$ related to a filter $G$ of $L$ and if $F \subseteq G$, then $\bar{\Theta}(F ; S) \subseteq \bar{\Psi}(G ; S), \forall S \in \mathcal{P}(L)$.

Proof. (1) is straightforward.

(2) For any subsets $S$ and $T$ of $L$, we have

$$
\begin{aligned}
x \in \bar{\Theta}(F ; S \cup T) & \Leftrightarrow F_{x} \cap(S \cup T) \neq \emptyset \\
& \left.\left.\Leftrightarrow(F)_{x} \cap S\right) \cup(F)_{x} \cap T\right) \neq \emptyset \\
& \Leftrightarrow F_{x} \cap S \neq \emptyset \text { or } F_{x} \cap T \neq \emptyset \\
& \Leftrightarrow x \in \bar{\Theta}(F ; S) \text { or } x \in \bar{\Theta}(F ; T) \\
& \Leftrightarrow x \in \bar{\Theta}(F ; S) \cup \bar{\Theta}(F ; T),
\end{aligned}
$$

and hence $\bar{\Theta}(F ; S \cup T)=\bar{\Theta}(F ; S) \cup \bar{\Theta}(F ; T)$.

(3) For any subsets $S$ and $T$ of $L$ we have

$$
\begin{aligned}
x \in \underline{\Theta}(F ; S \cap T) & \Leftrightarrow F_{x} \subseteq S \cap T \\
& \Leftrightarrow F_{x} \subseteq S \text { and } F_{x} \subseteq T \\
& \Leftrightarrow x \in \underline{\Theta}(F ; S) \text { and } x \in \underline{\Theta}(F ; T) \\
& \Leftrightarrow x \in \underline{\Theta}(F ; S) \cap \underline{\Theta}(F ; T) .
\end{aligned}
$$

Hence $\underline{\Theta}(F ; S \cap T)=\underline{\Theta}(F ; S) \cap \underline{\Theta}(F ; T)$.

(4) Let $S, T \in \mathcal{P}(L)$ be such that $S \subseteq T$. Then $S \cap T=S$ and $S \cup T=T$. It follows from (3) and (2) that

$$
\begin{aligned}
& \underline{\Theta}(F ; S)=\underline{\Theta}(F ; S \cap T)=\underline{\Theta}(F ; S) \cap \underline{\Theta}(F ; T), \\
& \bar{\Theta}(F ; T)=\bar{\Theta}(F ; S \cup T)=\bar{\Theta}(F ; S) \cup \bar{\Theta}(F ; T),
\end{aligned}
$$

which yields $\underline{\Theta}(F ; S) \subseteq \underline{\Theta}(F ; T)$ and $\bar{\Theta}(F ; S) \subseteq \bar{\Theta}(F ; T)$, respectively.

(5) Since $S \subseteq S \cup T$ and $T \subseteq S \cup T$, it follows from (4) that

$$
\underline{\Theta}(F ; S) \subseteq \underline{\Theta}(F ; S \cup T), \quad \underline{\Theta}(F ; T) \subseteq \underline{\Theta}(F ; S \cup T) .
$$

Thus $\underline{\Theta}(F ; S) \cup \underline{\Theta}(F ; T) \subseteq \underline{\Theta}(F ; S \cup T)$. 
(6) Since $S \cap T \subseteq S, T$, it follows from (4) that

$$
\bar{\Theta}(F ; S \cap T) \subseteq \bar{\Theta}(F ; S), \quad \bar{\Theta}(F ; S \cap T) \subseteq \bar{\Theta}(F ; T),
$$

so that $\bar{\Theta}(F ; S \cap T) \subseteq \bar{\Theta}(F ; S) \cap \bar{\Theta}(F ; T)$.

(7) If $x \in \bar{\Theta}(F ; S)$, then $F_{x} \cap S \neq \emptyset$, and so there exists $a \in S$ such that $a \in F_{x}$. Hence $(a, x) \in \Theta$, that is, $\{a \rightarrow x, x \rightarrow a\} \subseteq F$. Since $F \subseteq G$, it follows that $\{a \rightarrow x, x \rightarrow a\} \subseteq G$ so that $(a, x) \in \Psi$, that is, $a \in G_{x}$. Therefore $a \in G_{x} \cap S$, which means $x \in \bar{\Psi}(G ; S)$. This completes the proof.

Proposition 4.4. Let $\Theta$ be an equivalence relation on $L$ related to any filter $F$ of $L$. Then $\underline{\Theta}(F ; L)=L=\bar{\Theta}(F ; L)$, that is, $L$ is definable.

Proof. Straightforward.

Proposition 4.5. Let $\Theta$ be an equivalence relation on $L$ related to the trivial filter $\{1\}$ of $L$. Then $\underline{\Theta}(\{1\} ; S)=S=\bar{\Theta}(\{1\} ; S)$ for every nonempty subset $S$ of $L$, that is, every nonempty subset of $L$ is definable.

Proof. Note that $\{1\}_{x}=\{x\}$ for all $x \in L$, since if $a \in\{1\}_{x}$ then $(a, x) \in \Theta$ and hence $a \rightarrow x=1$ and $x \rightarrow a=1$. It follows that $a=x$. Hence

$$
\begin{aligned}
& \underline{\Theta}(\{1\} ; S)=\left\{x \in L \mid\{1\}_{x} \subseteq S\right\}=S, \\
& \bar{\Theta}(\{1\} ; S)=\left\{x \in L \mid\{1\}_{x} \cap S \neq \emptyset\right\}=S .
\end{aligned}
$$

This completes the proof.

Definition 4.6. Let $\Theta$ be an equivalence relation on $L$ related to a filter $F$ of $L$. A nonempty subset $S$ of $L$ is called an upper (resp. a lower) rough quasi-subalgebra/filter of $L$ if the upper (resp. nonempty lower) approximation of $S$ is a quasi-subalgebra/filter of $L$. If $S$ is both an upper and a lower rough quasi-subalgebra/filter of $L$, we say that $S$ is a rough quasi-subalgebra/filter of $L$.

THEOREM 4.7. Let $\Theta$ be an equivalence relation on $L$ related to a filter $F$ of $L$. Then every quasi-subalgebra $S$ of $L$ is a rough quasi-subalgebra of $L$.

Proof. Let $x, y \in \underline{\Theta}(F ; S)$. Then $F_{x} \subseteq S$ and $F_{y} \subseteq S$. Since $S$ is a quasi-subalgebra of $L$, it follows that $F_{x \rightarrow y}=F_{x} \rightsquigarrow F_{y} \subseteq S$ so that $x \rightarrow y \in$ $\underline{\Theta}(F ; S)$. Hence $\underline{\Theta}(F ; S)$ is a quasi-subalgebra of $L$. Now if $L, y \in \bar{\Theta}(F ; S)$, then $F_{x} \cap S \neq \emptyset$ and $F_{y} \cap S \neq \emptyset$, and so there exist $a, b \in S$ such that $a \in F_{x}$ and $b \in F_{y}$. It follows that $(a, x) \in \Theta$ and $(b, y) \in \Theta$. Since $\Theta$ is a congruence relation on $L$, we have $(a \rightarrow b, x \rightarrow y) \in \Theta$. Hence $a \rightarrow b \in F_{x \rightarrow y}$. Since $S$ is a quasi-subalgebra of $L$, we get $a \rightarrow b \in S$, and therefore $a \rightarrow b \in F_{x \rightarrow y} \cap S$, that is, $F_{x \rightarrow y} \cap S \neq \emptyset$. This shows that $x \rightarrow y \in \bar{\Theta}(F ; S)$, and consequently $\bar{\Theta}(F ; S)$ is a quasi-subalgebra of $L$. This completes the proof. 
Corollary 4.8. Let $\Theta$ be an equivalence relation on $L$ related to a filter $F$ of $L$. Then $\underline{\Theta}(F)(\neq \emptyset)$ and $\bar{\Theta}(F)$ are quasi-subalgebras of $L$, that is, $F$ is a rough quasi-subalgebra of $L$.

Proof. Straightforward.

THEOREM 4.9. Let $\Theta$ be an equivalence relation on $L$ related to a filter $F$ of $L$. If $U$ is a filter of $L$ containing $F$, then:

(1) $\underline{\Theta}(F ; U)(\neq \emptyset)$ is a filter of $L$, that is, $U$ is a lower rough filter of $L$.

(2) $\bar{\Theta}(F ; U)$ is a filter of $L$, that is, $U$ is an upper rough filter of $L$.

Proof. Let $U$ be a filter of $L$ containing $F$. Let $x \in F_{1}$. Then $x=1$ $\rightarrow x \in F \subseteq U$, and so $F_{1} \subseteq U$. Hence $1 \in \underline{\Theta}(F ; U)$. Let $x, y \in L$ be such that $x \in \underline{\Theta}(F ; U)$ and $x \rightarrow y \in \underline{\Theta}(F ; U)$. Then $F_{x} \subseteq U$ and $F_{x} \rightsquigarrow F_{y}=$ $F_{x \rightarrow y} \subseteq U$. Let $a \in F_{x}$ and $b \in F_{y}$. Then $(a, x) \in \Theta$ and $(b, y) \in \Theta$, which implies $(a \rightarrow b, x \rightarrow y) \in \Theta$. Hence $a \rightarrow b \in F_{x \rightarrow y} \subseteq U$. Since $a \in F_{x} \subseteq U$ and $U$ is a filter, it follows that $b \in U$ so that $F_{y} \subseteq U$. Thus $y \in \underline{\Theta}(F ; U)$. This shows that $\underline{\Theta}(F ; U)$ is a filter of $L$, that is, $U$ is a lower rough filter of $L$.

Now, obviously $1 \in \bar{\Theta}(F ; U)$. Let $L, y \in L$ be such that $x \in \bar{\Theta}(F ; U)$ and $x \rightarrow y \in \bar{\Theta}(F ; U)$. Then $F_{x} \cap U \neq \emptyset$ and $F_{x \rightarrow y} \cap U \neq \emptyset$, and so there exist $a, b \in U$ such that $a \in F_{x}$ and $b \in F_{x \rightarrow y}$. Hence $(a, x) \in \Theta$ and $(b, x \rightarrow y) \in \Theta$, which implies $a \rightarrow x \in F \subseteq U$ and $b \rightarrow(x \rightarrow y) \in F \subseteq U$. Since $a, b \in U$ and $U$ is a filter, we get $x \in U$ and $x \rightarrow y \in U$; hence $y \in U$. Note that $y \in F_{y}$, thus $y \in F_{y} \cap U$, that is, $F_{y} \cap U \neq \emptyset$. Therefore $y \in \bar{\Theta}(F ; U)$, and consequently $U$ is an upper rough filter of $L$.

Corollary 4.10. Let $\Theta$ be an equivalence relation on $L$ related to a filter $F$ of $L$. Then $\underline{\Theta}(F)(\neq \emptyset)$ and $\bar{\Theta}(F)$ are filters of $L$, that is, $F$ is a rough filter of $L$.

Theorem 4.9 shows that the notion of an upper (resp. a lower) rough filter extends the notion of a filter in a lattice implication algebra.

Let $f: L_{1} \rightarrow L_{2}$ be an implication homomorphism of lattice implication algebras. The dual kernel of $f$ is defined to be the set

$$
\operatorname{Dker}(f):=\left\{x \in L_{1} \mid f(x)=1\right\} .
$$

Obviously, $\operatorname{Dker}(f)$ is a filter of $L_{1}$.

THEOREM 4.11. If $f: L_{1} \rightarrow L_{2}$ is an implication homomorphism of lattice implication algebras, then

$$
\left(\forall G \in \mathcal{P}^{*}\left(L_{1}\right)\right)\left(f(\operatorname{Dker}(f) \rightarrow G)=f(G) \subseteq f\left(\varrho^{*}(G)\right)\right)
$$

where $\varrho$ is a congruence relation on $L_{1}$.

Proof. Let $G \in \mathcal{P}^{*}\left(L_{1}\right)$ and let $y \in f(G)$. Then $y=f(a)$ for some $a \in G$. Since $G \subseteq \varrho^{*}(G)$, it follows that $y=f(a) \in f\left(\varrho^{*}(G)\right)$. Since $1 \in \operatorname{Dker}(f)$, 
we have

$$
y=f(a)=f(1 \rightarrow a) \in f(\operatorname{Dker}(f) \rightarrow G)
$$

and so $f(G) \subseteq f(\operatorname{Dker}(f) \rightarrow G)$. Now if $z \in f(\operatorname{Dker}(f) \rightarrow G)$, then $f(x)=z$ for some $x \in \operatorname{Dker}(f) \rightarrow G$. Hence $x=a \rightarrow b$ for some $a \in \operatorname{Dker}(f)$ and $b \in G$. Then

$$
z=f(x)=f(a \rightarrow b)=f(a) \rightarrow f(b)=1 \rightarrow f(b)=f(b) \in f(G),
$$

and so $f(\operatorname{Dker}(f) \rightarrow G) \subseteq f(G)$. This completes the proof.

THEOREM 4.12. Let $f: L_{1} \rightarrow L_{2}$ be an implication homomorphism of lattice implication algebras. If $\Theta$ is a congruence relation on $L$ related to $\operatorname{Dker}(f)$, then $f(G)=f(\bar{\Theta}(\operatorname{Dker}(f) ; G))$ for every $G \in \mathcal{P}^{*}\left(L_{1}\right)$.

Proof. Using Theorem 4.11, we have $f(G) \subseteq f(\bar{\Theta}(\operatorname{Dker}(f) ; G))$ for all $G \in \mathcal{P}^{*}\left(L_{1}\right)$. Let $y \in f(\bar{\Theta}(\operatorname{Dker}(f) ; G))$. Then there exists $x \in \bar{\Theta}(\operatorname{Dker}(f) ; G)$ such that $y=f(x)$. Hence $\operatorname{Dker}(f)_{x} \cap G \neq \emptyset$, and so there exists $a \in G$ such that $a \in \operatorname{Dker}(f)_{x}$. Now $a \in \operatorname{Dker}(f)_{x}$ implies that $\{a \rightarrow x, x \rightarrow a\} \subseteq$ $\operatorname{Dker}(f)$. Thus

$$
f(a) \rightarrow f(x)=f(a \rightarrow x)=1, \quad f(x) \rightarrow f(a)=f(x \rightarrow a)=1 .
$$

It follows from (I4) that $f(a)=f(x)$ so that $y=f(x)=f(a) \in f(G)$. Therefore $f(\bar{\Theta}(\operatorname{Dker}(f) ; G)) \subseteq f(G)$, which completes the proof.

Acknowledgements. This work was supported by Korea Research Foundation Grant (KRF-2003-005-C00013), and was partially supported by the National Natural Science Foundation of P. R. China (Grant No. 60474022 ).

\section{References}

[1] R. Biswas and S. Nanda, Rough groups and rough subgroups, Bull. Polish Acad. Sci. Math. 42 (1994), 251-254.

[2] L. Bolc and P. Borowik, Many-Valued Logic, Springer, Berlin, 1994.

[3] W. A. Dudek, Y. B. Jun and H. S. Kim, Rough set theory applied to BCI-algebras, Quasigroups Related Systems 9 (2002), 45-54.

[4] J. A. Goguen, The logic of inexact concepts, Synthese 19 (1969), 325-373.

[5] Y. B. Jun, Implicative filters of lattice implication algebras, Bull. Korean Math. Soc. 34 (1997), 193-198.

[6] -, Fantastic filters of lattice implication algebras, Int. J. Math. Math. Sci. 24 (2000), $277-281$.

[7] —, On n-fold implicative filters of lattice implication algebras, ibid. 26 (2001), 695699.

[8] -, Roughness of ideals in BCK-algebras, Sci. Math. Jpn. 57 (2003), 165-169.

[9] Y. B. Jun, Y. Xu and K. Y. Qin, Positive implicative and associative filters of lattice implication algebras, Bull. Korean Math. Soc. 35 (1998), 53-61.

[10] S. Y. Kim, E. H. Roh and Y. B. Jun, On ultra filters of lattice implication algebras, Sci. Math. 2 (1999), 201-204. 
[11] N. Kuroki, Rough ideals in semigroups, Inform. Sci. 100 (1997), 139-163.

[12] N. Kuroki and J. N. Mordeson, Structure of rough sets and rough groups, J. Fuzzy Math. 5 (1997), 183-191.

[13] C. R. Lim and H. S. Kim, Rough ideals in BCK/BCI-algebras, Bull. Polish Acad. Sci. Math. 51 (2003), 59-67.

[14] J. Liu and Y. Xu, On certain filters in lattice implication algebras, Chinese Quart. J. Math. 11 (1996), 106-111.

[15] -, Filters and structure of lattice implication algebras, Chinese Sci. Bull. 42 (1997), $1517-1520$.

[16] V. Novak, First order fuzzy logic, Studia Logica 46 (1982), 87-109.

[17] J. Pavelka, On fuzzy logic I, II, III, Z. Math. Logik Grundlag. Math. 25 (1979), 45-52, 119-134, 447-464.

[18] Z. Pawlak, Rough sets, Int. J. Comput. Inform. Sci. 11 (1982), 341-356.

[19] -, Rough Sets. Theoretical Aspects of Reasoning about Data, Kluwer, Dordrecht, 1991.

[20] F. Wang, Modeling analysis and synthesis of linguistic dynamic systems: a computational theory, in: Proc. IEEE Internat. Workshop on Architecture for Semiotic Modeling and Situation Control in Large Complex Systems, Monterery, CA, 27-30 August 1995, 173-178.

[21] -, Outline of a computational theory for linguistic dynamic systems: toward computing with words, Int. J. Intell. Control Systems 2 (1998), 211-224.

[22] Y. Xu, Homomorphisms in lattice implication algebras, in: Proc. 5th Many-Valued Logical Congress of China, 1992, 206-211.

[23] —, Lattice implication algebras, J. Southwest Jiaotong Univ. 1 (1993), 20-27.

[24] Y. Xu and K. Qin, On filters of lattice implication algebras, J. Fuzzy Math. 1 (1993), 251-260.

[25] Y. Xu, D. Ruan, K. Qin and J. Liu, Lattice-Valued Logic, Springer, Berlin, 2003.

[26] L. A. Zadeh, Fuzzy sets, Inform. Control 8 (1965), 338-353.

[27] - , The concept of a linguistic variable and its application to approximate reasoning, I, II, III, Inform. Sci. 8 (1975), 199-249, 8 (1975), 301-357, 9 (1975), 43-80.

[28] L. A. Zadeh, Fuzzy logic = computing with words, IEEE Trans. Fuzzy Systems 4 (1996), 103-111.

Young Bae Yun

Department of Mathematics Education

Gyeongsang National University

Chinju 660-701, Korea

E-mail: ybjun@gsnu.ac.kr icedoor@hotmail.com
Yang $\mathrm{Xu}$

Department of Applied Mathematics Southwest Jiaotong University Chungdu, Sichuan 610031, China E-mail: xuyang@swjtu.edu.cn 